\title{
Yarn Diameter and Linear Mass Correlation
}

\author{
Vítor Carvalho · Rosa M. Vasconcelos • \\ Filomena O. Soares $•$ Michael Belsley
}

Published online: 2 May 2009

(C) Springer Science+Business Media, LLC 2009

\begin{abstract}
This paper focuses on the determination of the statistical correlation between yarn diameter and yarn linear mass. The experimental methods employed are based on optical analysis and on image processing techniques applied to electron microscope images. Several different cotton yarns were examined over a wide range of yarn linear masses. The results indicate that diameters predicted by the relationship commonly quoted in the literature can be as much as $62 \%$ smaller than those experimentally observed.
\end{abstract}

Keywords Yarn diameter - Cotton yarn - Experimental . Linear mass

\section{Introduction}

A key parameter in the textile industry is the relation between a yarn's diameter and its linear mass. For example, capacitive measurements are often used to characterize a given yarn's linear mass and from this the average diameter is deduced. If one assumes that the yarn has, on average,

V. Carvalho ( $\varangle) \cdot$ F.O. Soares

DEI, UM, Azurém, Guimarães, Portugal

e-mail: vcarvalho@dei.uminho.pt

F.O. Soares

e-mail: fsoares@dei.uminho.pt

R.M. Vasconcelos

DET, UM, Azurém, Guimarães, Portugal

e-mail: rosa@det.uminho.pt

M. Belsley

DF, UM, Gualtar, Braga, Portugal

e-mail: belsley@ fisica.uminho.pt a cylindrical shape it is clear from simple geometrical considerations that the yarn diameter should be proportional to the square root of its linear mass. Following the industry standard of expressing linear mass in $\mathrm{g} / \mathrm{km}$, call tex, one can immediately arrive at the following expression $[1,2]$

$$
\begin{gathered}
\operatorname{tex}(\mathrm{g} / \mathrm{km})=\phi \times \rho\left(\mathrm{g} / \mathrm{cm}^{3}\right) \times \pi\left(\frac{d(\mathrm{~cm})}{2}\right)^{2} \times\left(\frac{10^{5} \mathrm{~cm}}{\mathrm{~km}}\right) \\
\Rightarrow d(\mathrm{~cm})=\sqrt{4 \times \operatorname{tex} /\left(\pi \times 10^{5} \times \phi \times \rho\right)} .
\end{gathered}
$$

Here $\phi$ represents the yarn "porosity", which is the fraction of the yarn actually occupied by fibers, the rest being air. It is curious that most literature values result from theoretical considerations, assuming that the individual fibers are themselves cylindrical and closely packed within the yarn. To our knowledge there does not seem to be any experimental confirmation of these assumptions. Theoretical literature values for the porosity typically range from 0.55 to 0.70 [2-5]. If we take as a characteristic value for the porosity the median value of 0.63 then for $100 \%$ cotton yarns $\left(\rho=1.5 \mathrm{~g} / \mathrm{cm}^{3}\right)$, the expected relation between diameter and linear mass density is

$d(m m)=0.037 \sqrt{\text { tex }}$.

Here we have included a simple units conversion and expressed the diameter in millimeters instead of centimeters as in Eq. 1. This is the relation commonly encountered in the literature. While the square-root dependence on linear mass is simply a consequence of the assumed cylindrical geometry, the appropriate value of porosity is not at all obvious. In an effort to experimentally determine this parameter, we have analyzed several different cotton yarns with linear 
masses ranging from 4.2 to 295 tex and fit the results to an equation of the general form,

$d(m m)=K \sqrt{t e x}$.

Our results show that the typical literature values, $K=$ 0.037 , are $62 \%$ lower than our experimental findings. This suggests that the actually porosity parameter, $\phi$, is roughly a third of what is typically assumed. While this seems intuitively to be extremely low, we have acquired electron microscope images which suggest that the actual volume occupied by cotton fibers is in fact quite low. In the following section we briefly describe the experimental analysis [6, 7] used to deduce the relation between yarn linear mass and diameter. Our experimental technique is in principle capable of determining diameters with a precision of a few microns.

\section{Experimental Procedure}

The main goal of the study was to experimentally obtain the correlation between yarn mass and yarn diameter validating Eq. 3 and in the process to experimentally determine the mean porosity factor of Eq. 1. The optical experimental set-up used in this study is shown in Fig. 2.

The main purpose of the optical setup is to produce an image that can be analyzed to provide information regarding the local degree of yarn hairiness (i.e. the amount of fibers which protrude significantly from the yarn core, as seen in Fig. 1) [8] and yarn core diameter in the final image plane (position of the photo detector in Fig. 2) (I/PD). Coherent light from the laser diode in a single transverse mode is incident on a collimating lens (L1) and directed to the yarn, placed in the object holder (O). The size of the final image detected by the photodiode array (I/PD) is controlled by the lenses L2 and L3.

A custom spatial filter with roughly $1 \mathrm{~mm}$ of opaque target $(\mathrm{F})$ placed in the Fourier plane of L2, blocks all spatial frequencies below $10 \mathrm{~mm}^{-1}$ (high pass spatial Fourier filter), corresponding to a characteristic size of 100 microns or larger in the object plane. Textile yarns typically have diameters ranging from a few microns up to millimeters, while the small hairs protruding from the yarn are single fibers with diameters typically less than 10 microns [9]. As there is a clear separation in the characteristic length scales of the yarns, this results in the contours of the edges of the yarn and associated hairs being highlighted while simultaneously eliminating the constant background illumination of the incident laser beam (Fig. 3).

Figure 4 shows the line profile result of a linear section of this image along the line profile highlighted in Fig. 3. Our actual data was acquired using a linear photodiode array (S8378-256Q from Hamamatsu, with a pitch of $25 \mu \mathrm{m}$ ) in the image plane. These devices are consist of multiple photo-sensitive areas (pixels), organized over a single line,

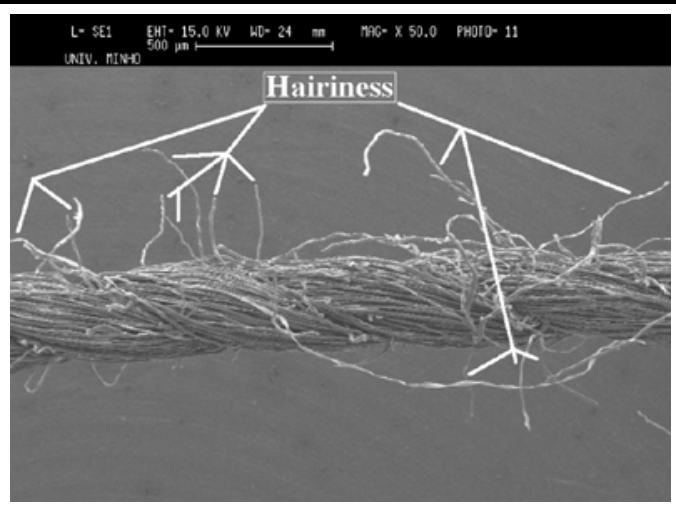

Fig. 1 Identification of yarn hairiness in an electron microscope photograph

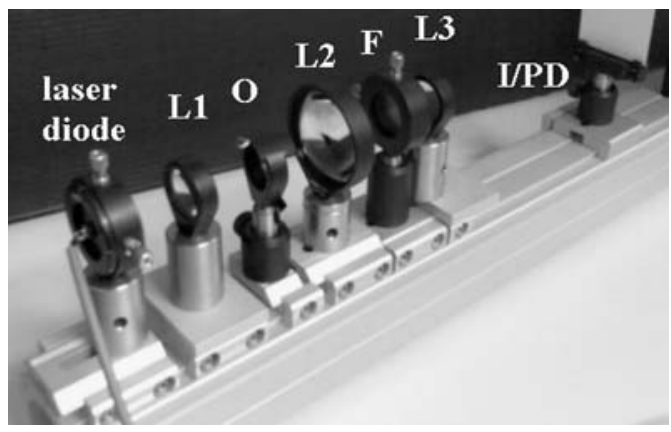

Fig. 2 Custom developed optical yarn measurement hardware

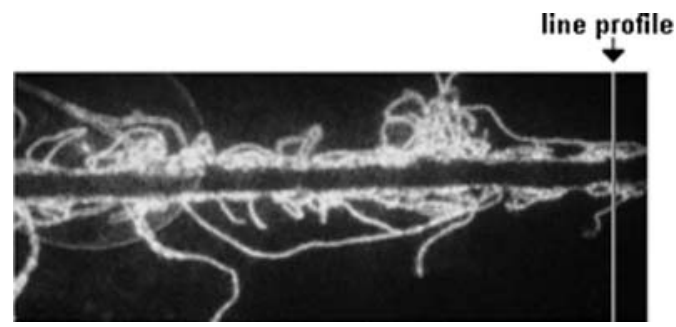

Fig. 3 Example of an image resulting from coherent optical signal processing

which convert a spatial light distribution to a proportional current or voltage signal distributed sequentially in time. The yarn diameter in the image plane corresponds to the difference of pixels between the peak positions of the right and left yarn contours, multiplied by the pitch of each pixel. This can then be easily converted to obtain the actual yarn diameter in the object plane using the measured optical system magnification.

In addition, to increase the amount of available data, image processing techniques were applied to previously acquired electron microscope images. These images were digitized and the number of pixels between yarn contours on opposite sides of the yarn was determined. Using a similar technique for the magnification calibration bar of each image, the actual yarn diameter could easily be found with a 
Fig. 4 Red plane line profile obtained for Fig. 2 with 512 pixels

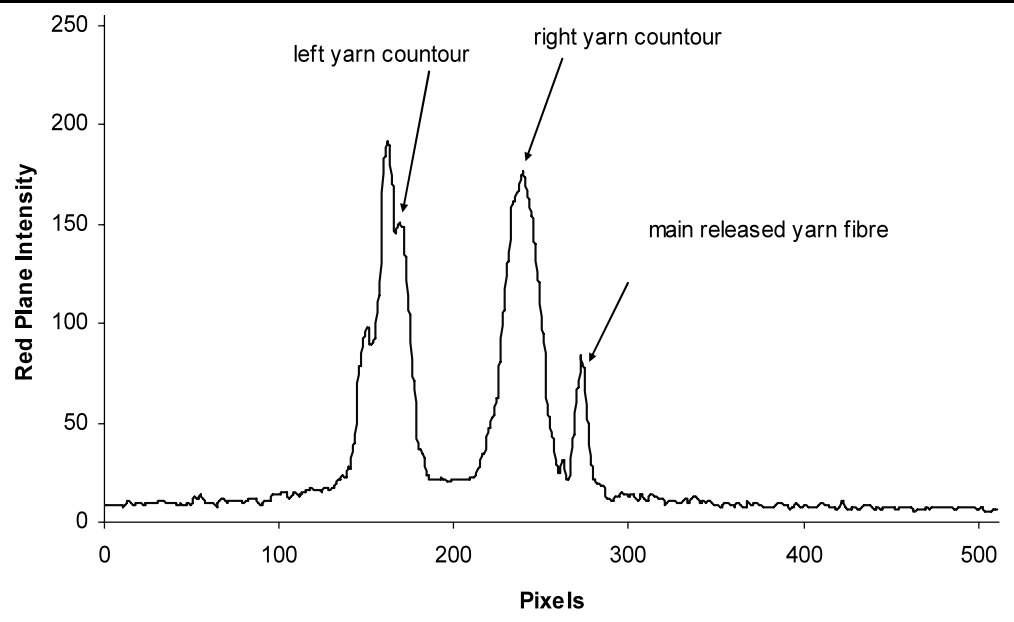

Pixels

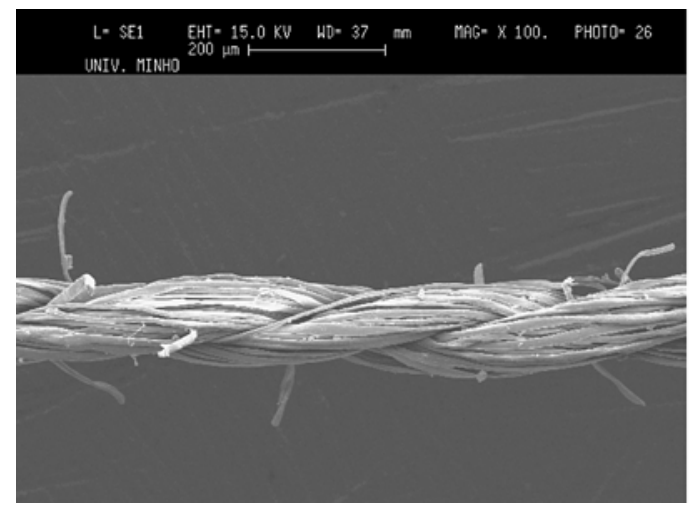

Fig. 5 Pictures obtained with the electron microscope for yarns 4.20 tex (left photograph) (folded or multi-cable yarn [10], two cables, anti-clockwise yarn orientation) and 48.44 tex (right photograph) (un-

precision of order of a few microns or better, depending on the magnification of the electron microscope image.

Several different analyses were performed:

- Four different $100 \%$ cotton yarns (19.67 tex, 36.88 tex, 62.00 tex and 295.00 tex), with an optical diameter characterization, using ten different sample positions for each yarn;

- Four different $100 \%$ cotton yarns $(4.20$ tex, 48.44 tex, 75.10 tex and 212.20 tex), with a diameter characterization based on image processing under electron microscope images, using also ten different locations for each yarn.

The pictures obtained using the electron microscope for the 4.20 tex and 48.44 tex yarns are shown in Fig. 5, while those for the 75.10 tex and 212.20 tex yarns are displayed in Fig. 6.

\section{Results}

The results of the various diameter measurements are summarized in Table 1. For each yarn analyzed ten individ-

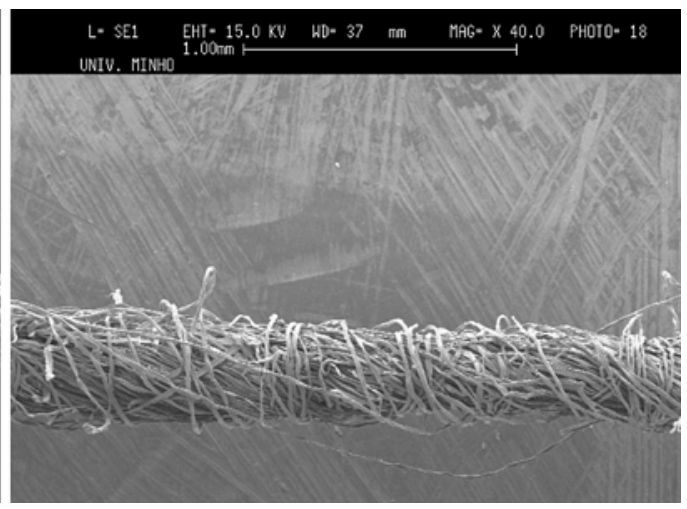

folded, spun or single cable yarn [11], one cable, clockwise fiber orientation) from which were considered individually ten different sections for average diameter characterization

ual diameter measurements were carried out. Based on a nonparametric test the two procedures used in the diameter measurement (IMP, Image Procedure Measurement, and OM, Optical Measurement) are not statistically significant $(p>0.05)$ [12]. As a first step we thought it was important to fit simultaneously both the proportionality constant $\mathrm{K}$ and the power, a, of the linear mass (tex) in Eq. 4.

$d(m m)=K \cdot t e x^{a}$.

Figure 7 shows the results obtained employing a power dependence, where the best fit parameters determined to Eq. 4, where $K=0.062 \pm 0.002$ and $a=0.490 \pm 0.009$, with $R^{2}=0.973$.

Furthermore, we also tested the linearization of the power correlation model, where new adjustment parameters were calculated [12]. However, as the two models did not show significant differences and considering the parallelism to the theoretical literature model used as a reference for this study, the power correlation model was applied.

The results shown graphically in Fig. 7 confirm that the power law dependence is the expected result of $1 / 2$ for 


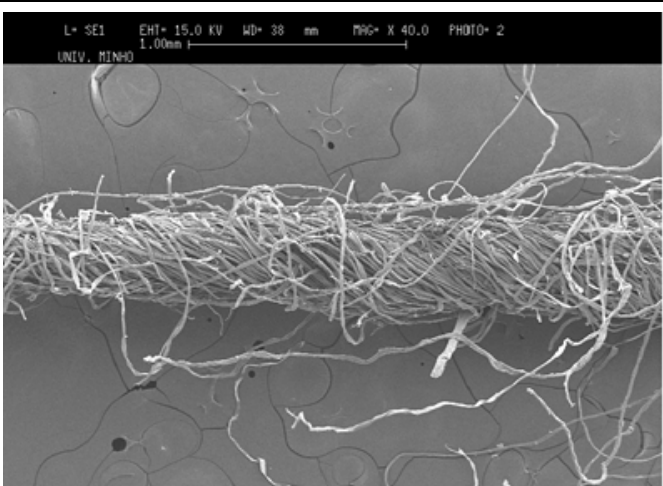

Fig. 6 Pictures obtained with the electron microscope for yarns 75.10 tex (left photograph) (unfolded yarn [11], one cable, clockwise fiber orientation), and 212.20 tex (right photograph) (folded yarn [10], four

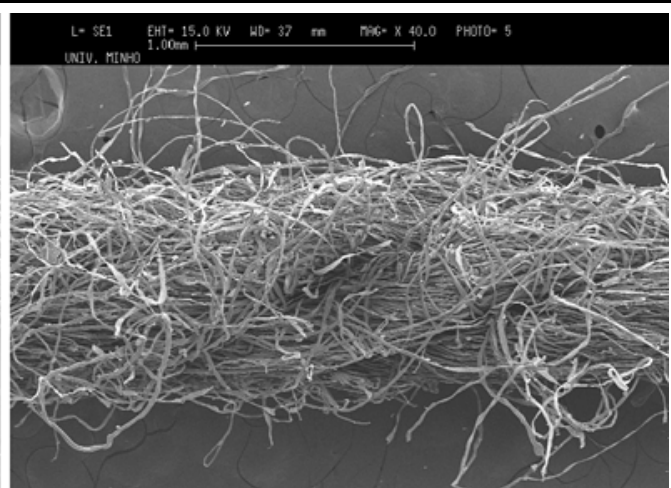

cables, anti-clockwise yarn orientation). Ten different diameters were determined from separate positions along the fibers in these images

Table 1 Experimental diameter and constant $K$ results

\begin{tabular}{|c|c|c|c|c|c|c|c|c|c|c|c|c|c|}
\hline $\begin{array}{l}\text { Yarn } \\
\text { (tex) }\end{array}$ & $\begin{array}{l}\text { Sample } \\
1\end{array}$ & $\begin{array}{l}\text { Sample } \\
2\end{array}$ & $\begin{array}{l}\text { Sample } \\
3\end{array}$ & $\begin{array}{l}\text { Sample } \\
4\end{array}$ & $\begin{array}{l}\text { Sample } \\
5\end{array}$ & $\begin{array}{l}\text { Sample } \\
6\end{array}$ & $\begin{array}{l}\text { Sample } \\
7\end{array}$ & $\begin{array}{l}\text { Sample } \\
8\end{array}$ & $\begin{array}{l}\text { Sample } \\
9\end{array}$ & $\begin{array}{l}\text { Sample } \\
10\end{array}$ & $\begin{array}{l}\text { Average } \\
(\mathrm{mm})\end{array}$ & $\begin{array}{l}\mathrm{SD} \\
(\mathrm{mm})\end{array}$ & $\begin{array}{l}K \\
\text { (experimental) }\end{array}$ \\
\hline 4.20 & 0.11 & 0.12 & 0.13 & 0.13 & 0.14 & 0.14 & 0.13 & 0.14 & 0.15 & 0.14 & 0.13 & \pm 0.01 & 0.065 \\
\hline 19.67 & 0.25 & 0.25 & 0.21 & 0.32 & 0.32 & 0.25 & 0.25 & 0.32 & 0.28 & 0.29 & 0.27 & \pm 0.04 & 0.062 \\
\hline 36.88 & 0.32 & 0.39 & 0.36 & 0.36 & 0.32 & 0.29 & 0.36 & 0.32 & 0.36 & 0.39 & 0.35 & \pm 0.03 & 0.057 \\
\hline 48.44 & 0.45 & 0.43 & 0.41 & 0.36 & 0.37 & 0.32 & 0.32 & 0.36 & 0.4 & 0.38 & 0.38 & \pm 0.04 & 0.055 \\
\hline 62.00 & 0.46 & 0.54 & 0.43 & 0.43 & 0.43 & 0.46 & 0.46 & 0.43 & 0.5 & 0.43 & 0.46 & \pm 0.04 & 0.058 \\
\hline 75.10 & 0.51 & 0.51 & 0.46 & 0.50 & 0.49 & 0.48 & 0.47 & 0.48 & 0.50 & 0.49 & 0.49 & \pm 0.02 & 0.056 \\
\hline 212.20 & 0.89 & 0.90 & 0.90 & 0.85 & 0.86 & 0.88 & 0.89 & 0.89 & 0.96 & 0.89 & 0.89 & \pm 0.03 & 0.061 \\
\hline 295.00 & 1.04 & 1.25 & 1 & 1.07 & 0.96 & 1 & 1.07 & 1 & 1.14 & 1.21 & 1.07 & \pm 0.10 & 0.063 \\
\hline
\end{tabular}

Image processing measurements

Optical measurements (photodiode array)

$K$ average $=0.060$

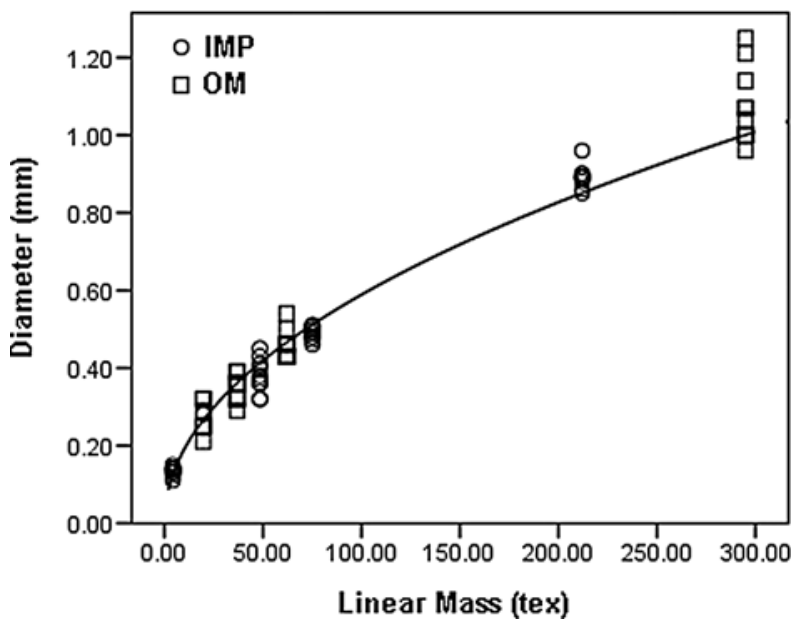

Fig. 7 Fit to the experimental data of Table 1, where the solid line represents the best fit obtained to Eq. 4

cylindrical yarns. Given the strong physical basis for the square root dependence on linear mass (tex) we have subse- quently, assumed this as given and focused attention on obtaining the best experimental fit to the proportionality constant $K$.

\subsection{Experimental Determination of the Constant $K$ Value}

As a first step the average diameter values for each yarn in Eq. 3 were used to determine an "experimental $K$ value" for each of the yarns analyzed. These values are reported in the final column of Table 1 . The average of the 8 experimental $K$ values is 0.060 .

Figure 8 presents the best fit to the experimental data assuming the functional form of Eq. 3. The resulting proportionality constant, $K=0.060 \pm 0.001$ with $R^{2}=0.972$, is quite close to that found above (Fig. 7) and similar to that given by the average of the "experimental $\mathrm{K}$ values" in Table 1.

Based on these results, there is little doubt that the experimentally determined correlations point to a proportionality constant, $K=0.060$ in Eq. 3. This value fits the experimen- 
Table 2 Comparative analysis between the theoretical and experimental diameters

\begin{tabular}{rlllll}
\hline $\begin{array}{l}\text { Yarn } \\
(\text { tex })\end{array}$ & $\begin{array}{l}\text { Experimental } K \\
\text { diameter } \\
\left(d_{e}\right)\end{array}$ & $\begin{array}{l}\text { Theoretical } K \\
\text { diameter } \\
(\mathrm{mm})\end{array}$ & $\begin{array}{l}\left.\Delta d=d_{e}-d_{t}\right) \\
(\mathrm{mm})\end{array}$ & $\begin{array}{l}\Delta d \\
(\mathrm{~mm})\end{array}$ & $\begin{array}{l}\text { Average diameter } \\
(\%)\end{array}$ \\
\hline 4.20 & 0.122963 & 0.07583 & 0.047136 & 61.67 & $\begin{array}{l}\text { Table 1) }-d_{e} \\
(\mathrm{~mm})\end{array}$ \\
19.67 & 0.266105 & 0.16410 & 0.102007 & & 0.007037 \\
36.88 & 0.364373 & 0.22470 & 0.139676 & & 0.003895 \\
48.44 & 0.417593 & 0.25752 & 0.160077 & & -0.014370 \\
62.00 & 0.472440 & 0.29134 & 0.181102 & & -0.037590 \\
75.10 & 0.519962 & 0.32064 & 0.199319 & & -0.012440 \\
212.20 & 0.874025 & 0.53898 & 0.335043 & & 0.015975 \\
295.00 & 1.030534 & 0.63550 & 0.395038 & & 0.039466 \\
\hline
\end{tabular}

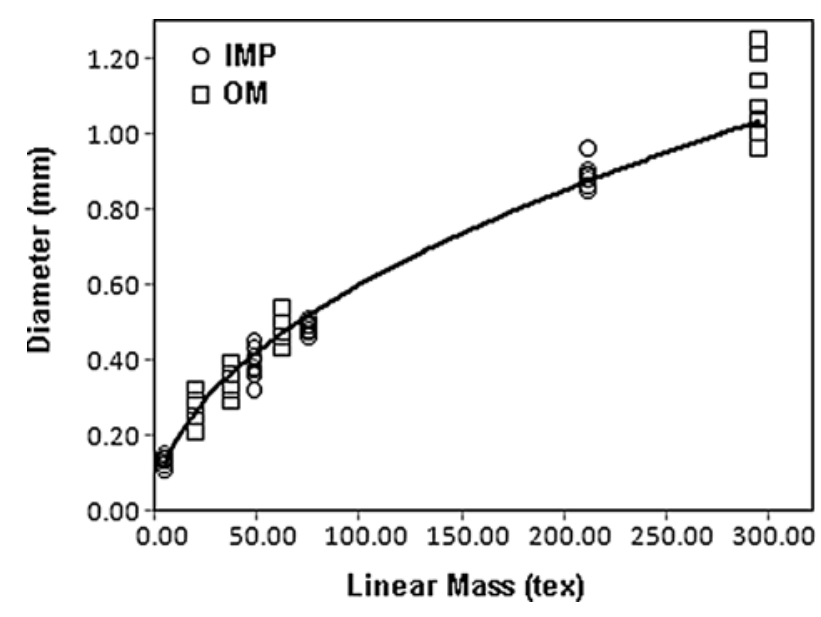

Fig. 8 Fit to the experimental data of Table 1, where the solid line represents the best fit obtained to Eq. 3

tal data for a wide range of tex values stretching from 4.2 up to 295 tex.

\subsection{Experimental and Theoretical Diameter Values}

Table 2 presents the diameter values obtained by using Eq. 3, considering the theoretical $K$ value found in the literature (0.037) and the experimental $K$ value (0.060).

Analyzing Table 2 , the theoretically predicted diameters are approximately $62 \%$ lower than the diameters determined with the experimental $K$ value. Moreover, the difference between the average diameters (Table 1 ) and the diameters determined using the experimental $K$ value (Table 2) is minimal and, for almost all yarns, within the standard deviation (SD) range of the diameters measurements (Table 1), as shown in Table 2.

Figure 9 illustrates the distribution curves of the theoretical and experimental diameter values. It can be observed that, as expected, the theoretical diameter results are always inferior to the experimental results.

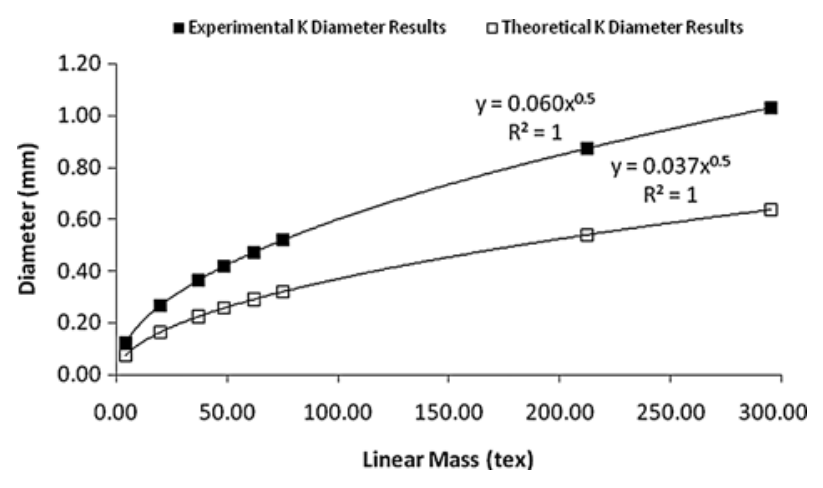

Fig. 9 Comparison of the experimental K results with those predicted by the theoretical relationship reported in the literature

As stated in the introduction, the theoretical literature values typically assume cylindrical fibers put together in a closed packed geometry. The obtained experimental results call into question this assumption. Indeed, if the experimental $K$ values are translated into an effective porosity, using Eq. 1, it is found that $100 \%$ cotton yarns have a porosity of only 0.232 , being quite low when compared to the theoretical values referred in the literature (between 0.55 and 0.70 ).

To lend additional support to this interpretation we have acquired electron microscope images of a transverse section of a 75.10 tex $100 \%$ cotton yarn. These images, presented in Fig. 10, show that there is a large amount of empty space in these yarns. Also observing other transversal pictures found in the literature $[13,14]$, the same phenomenon was verified. In addition the individual cotton fibers are far from cylindrical in shape, having a transverse profile that is more ribbon like. The $500 \times$ magnification image appears to be roughly consistent with the conclusion that only $23 \%$ of the yarn volume is actually occupied by cotton fibers.

\section{Conclusions and Future Work}

Using two different and independent experimental techniques we have shown that the commonly quoted relation- 

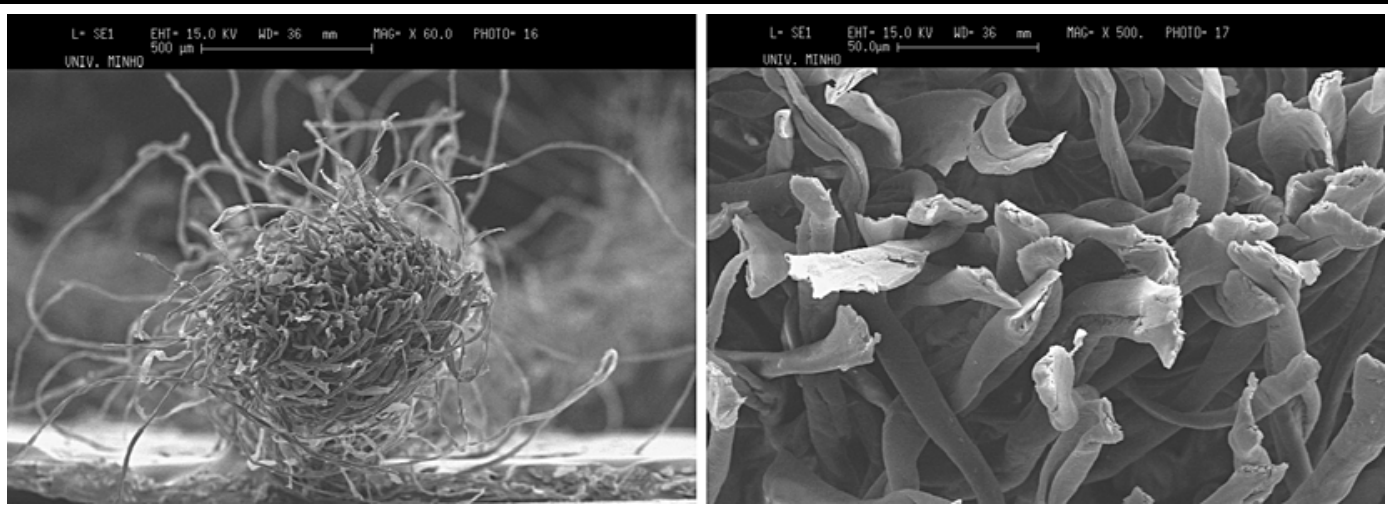

Fig. 10 Transversal analysis of the 75.10 tex yarn for a magnitude of $60 \times$ and $500 \times$

ship in the literature for diameter in function of the linear mass is in error by roughly $62 \%$ for the $100 \%$ cotton fiber analyzed. It would be interesting to discover if similar discrepancies exist for yarns made from other types of fibers. Indeed we plan to investigate this question in the near future.

Moreover, this study also shows that the diameters inferred from the capacitance measurement are in danger of being inaccurate if not properly calibrated by an appropriate porosity. This is because the capacitance measurements are sensitive only to the quantity of cotton fibers between the sensor plates and not to the empty space (air) between the fibers that constitute the yarn. There is therefore a strong need to accurately characterize the porosities of the various types of yarn commonly used in the textile industry.

Acknowledgements The authors are grateful to the Portuguese Foundation (FCT) for funding through the scholarship (BD/ 19028/ 2004).

\section{References}

1. http://business.vsnl.com/balasubramanian/YarnCloth.html\#DC (accessed in November 2008)
2. Castro, M., Araújo, M.: Manual de Engenharia Têxtil, vol. II. Gulbenkian, Lisbon (1986)

3. Hamilton, J.B.: J. Tex. Inst. 50, T.655 (1959)

4. Van Issun, B.E., Chamberlain, N.H.: J. Tex. Inst. 50, T.579 (1959)

5. Grosberg, P.: J. Tex. Inst. 57, T.383 (1966)

6. Carvalho, V., Belsley, M., Vasconcelos, R., Soares, F.: YSQ-high precision low cost system for yarn quality assessment. In: Engenharias '07-Inovação \& Desenvolvimento, Covilhã, Portugal, 2123 November 2007

7. Carvalho, V., Belsley, M., Vasconcelos, R., Soares, F.O.: Yarn hairiness and diameter characterization using a CMOS line array. Measurement 41(10), 1077-1092 (2008)

8. Barella, A.: The hairiness of yarns. Tex. Prog. 24(3) (1992)

9. Carvalho, V., Cardoso, P., Belsley, M., Vasconcelos, R., Soares, F.O.: Yarn hairiness characterization using two orthogonal directions. IEEE Trans. Instrum. Meas. 58(3), 594-601 (2009)

10. Rosiak, D., Przybyl, K.: Twisting of multi-folded yarns and threads manufactured by means of new spinning technologies. AUTEX Res. J. 4(3), 113-117 (2004)

11. Basal, G., Oxenham, W.: Vortex spun yarn vs. air-jet spun yarn. AUTEX Res. J. 3(3), 96-101 (2003)

12. Devore, J.: Probability and Statistics for Engineering and the Sciences, 7th edn. Duxbury, San Luis Obispo (2008)

13. Goswami, B.C., Martindale, J.G., Scardino, F.L.: Textile Yarns: Technology, Structure and Applications, 2nd edn. Wiley, New York (1977)

14. Kupper, J.R.: Tex. Res. J. 31, 490 (1961) 